hep-ph/9907377

UWThPh-1999-44

HEPHY-PUB 717/99

October 1, 2018

\title{
Large Higgs Boson Exchange Contribution in Three-Body Neutralino Decays
}

\author{
A. Bartl ${ }^{1}$, W. Majerotto ${ }^{2}$, and W. Porod ${ }^{3}$ \\ ${ }^{1}$ Institut für Theoretische Physik, Univ. Wien, A-1090 Vienna, \\ Austria \\ ${ }^{2}$ Institut für Hochenergiephysik der Österreichischen \\ Akademie der Wissenschaften, A-1050, Vienna, Austria \\ ${ }^{3}$ Departament de Física Teòrica-IFIC, CSIC, Univ. de \\ València Burjassot, València 46100, Spain
}

\begin{abstract}
We show that the Higgs boson exchange contribution can be large in three-body decays of neutralinos even in the case of small $\tan \beta$. This enlarges the branching ratios for the decays $\tilde{\chi}_{2}^{0} \rightarrow \tilde{\chi}_{1}^{0} b \bar{b}$ and $\tilde{\chi}_{2}^{0} \rightarrow \tilde{\chi}_{1}^{0} \tau^{-} \tau^{+}$. This is the case in the region of the parameter space where the two lightest neutralinos are gaugino-like, the sfermions are heavier than $300 \mathrm{GeV}$, and $m_{A^{0}} \lesssim 200 \mathrm{GeV}$.
\end{abstract}




\section{Introduction}

In the phenomenology of supersymmetric (SUSY) particles the neutralinos $\tilde{\chi}_{i}^{0}$ play a special rôle. In the Minimal Supersymmetric Standard Model (MSSM) one has four neutralinos $\tilde{\chi}_{i}^{0}, i=1, \ldots, 4$. If R-parity is conserved $\tilde{\chi}_{1}^{0}$ is considered to be the lightest stable SUSY particle (LSP). In $e^{+} e^{-}$or $p \bar{p}$ $(p p)$ collisions neutralinos $\tilde{\chi}_{i}^{0}$ as well as charginos $\tilde{\chi}_{j}^{+}, j=1,2$, are produced directly or in cascade decays of heavier SUSY particles. Therefore, a precise and complete theoretical knowledge of the decay properties of neutralinos and charginos is mandatory for the understanding of all aspects of SUSY phenomenology.

Neutralino decays have been extensively studied in the literature [1]. In the following we reexamine the decays of $\tilde{\chi}_{2}^{0}$. If two-body decays like $\tilde{\chi}_{2}^{0} \rightarrow$ $Z^{0} \tilde{\chi}_{1}^{0}, \tilde{\chi}_{2}^{0} \rightarrow h^{0} \tilde{\chi}_{1}^{0}, \tilde{\chi}_{2}^{0} \rightarrow f \tilde{\tilde{f}}$ (with $\tilde{f}$ being a slepton, sneutrino or squark) are kinematically not possible, the three-body decays

$$
\tilde{\chi}_{i}^{0} \rightarrow \tilde{\chi}_{j}^{0} f \bar{f}
$$

and

$$
\tilde{\chi}_{i}^{0} \rightarrow \tilde{\chi}_{j}^{+} f \bar{f}^{\prime}
$$

will dominate. Here $f\left(f^{\prime}\right)$ denote a Standard Model (SM) fermion. The decay (四) proceeds at tree level via $Z^{0}, \tilde{f}$ and Higgs boson exchange $\left(h^{0}, H^{0}\right.$, $A^{0}$ in the MSSM).

Usually, the Higgs boson exchange is assumed to be small or even negligible, at least for small or moderate $\tan \beta$, where $\tan \beta=v_{2} / v_{1}, v_{1,2}$ being the vacuum expectation values of the neutral Higgs fields in the MSSM. In this note we will point out that Higgs exchange can be important in a certain domain of the SUSY parameters for $\tilde{\chi}_{2}^{0} \rightarrow \tilde{\chi}_{1}^{0} b \bar{b}$ and $\tilde{\chi}_{2}^{0} \rightarrow \tilde{\chi}_{1}^{0} \tau^{-} \tau^{+}$. This 
is the case if $\tilde{\chi}_{1}^{0}$ and $\tilde{\chi}_{2}^{0}$ are gaugino-like $(M<|\mu|)$ and the sfermions are relatively heavy $\left(m_{\tilde{f}} \gtrsim 300 \mathrm{GeV}\right)$. In this case the $Z^{0}$ contribution will be suppressed because the $Z^{0}$ couples only to the higgsino components of $\tilde{\chi}_{1,2}^{0}$. The sfermion exchange contribution will also be suppressed due to the heavy sfermion mass. Then the Higgs boson exchange becomes important in the decays $\tilde{\chi}_{2}^{0} \rightarrow \tilde{\chi}_{1}^{0} b \bar{b}$ and $\tilde{\chi}_{2}^{0} \rightarrow \tilde{\chi}_{1}^{0} \tau^{-} \tau^{+}$due to the corresponding Yukawa couplings which are proportional to $m_{b}$ and $m_{\tau}$, respectively. This enlarges the branching ratios for these decays, even for small $\tan \beta$, and reduces the branching ratios for final states containing leptons and quarks of the first and second generation. For large $\tan \beta$ this lepton non-universality effect was already discussed in [2].

Moreover, the Higgs exchange contribution depends on the parameters of the Higgs sector. In the MSSM the $h^{0}-b-\bar{b}$ coupling depends on the Higgs mixing angle $\sin \alpha$ [3]. We will show that Higgs boson exchange plays a rôle at small $\tan \beta$ for $m_{A^{0}} \lesssim 200 \mathrm{GeV}$. For larger values of $m_{A^{0}}|\sin \alpha|$ becomes smaller and, therefore the Higgs boson exchange contribution becomes less important. The Higgs boson exchange has, of course, implications for the neutralino search at present and future colliders. For instance, the neutralino search at LEP [4, 5] and the trilepton signal at TEVATRON due to $p \bar{p} \rightarrow$ $\tilde{\chi}_{1}^{ \pm} \tilde{\chi}_{2}^{0} \rightarrow 3 l+p_{\text {Tmiss }}$ [6, 7] would be affected by the Higgs boson contributions in the decays.

\section{Neutralino Decays}

In the following we are interested in the decays Eq. (11) of $\tilde{\chi}_{2}^{0}$ for the case $|M| \ll|\mu|$. Then $\tilde{\chi}_{2}^{0}$ decays mainly into $\tilde{\chi}_{1}^{0} f \bar{f}$ because $m_{\tilde{\chi}_{2}^{0}} \approx 2 m_{\tilde{\chi}_{1}^{0}} \approx$ $m_{\tilde{\chi}_{1}^{+}} \approx M$. The main contributions stem from the $Z$-boson and Higgs boson 
exchanges if the sfermions are relatively heavy $\left(m_{\tilde{f}} \gtrsim 300 \mathrm{GeV}\right)$. For the following considerations it is instructive to have a look at the amplitudes of these contributions:

$$
\begin{aligned}
M_{Z} & =-g^{2} D_{Z}^{\mu \nu} O_{j i}^{L \prime \prime} \bar{u}\left(p_{l}\right) \gamma^{\mu}\left(L_{f} P_{L}+R_{f} P_{R}\right) v\left(p_{k}\right) \bar{u}\left(p_{j}\right) \gamma^{\nu} \gamma_{5} u\left(p_{i}\right), \\
M_{h^{0}} & =g^{2} D_{h^{0}} a_{j i}^{h^{0}} a_{f}^{h^{0}} \bar{u}\left(p_{j}\right) u\left(p_{i}\right) \bar{u}\left(p_{l}\right) v\left(p_{k}\right), \\
M_{H^{0}} & =g^{2} D_{H^{0}} a_{j i}^{H^{0}} a_{f}^{H^{0}} \bar{u}\left(p_{j}\right) u\left(p_{i}\right) \bar{u}\left(p_{l}\right) v\left(p_{k}\right), \\
M_{A^{0}} & =-g^{2} D_{A^{0}} a_{j i}^{A^{0}} a_{f}^{A^{0}} \bar{u}\left(p_{j}\right) \gamma_{5} u\left(p_{i}\right) \bar{u}\left(p_{l}\right) \gamma_{5} v\left(p_{k}\right),
\end{aligned}
$$

where $P_{L, R}=\left(1 \mp \gamma_{5}\right) / 2$ and $p_{i}, p_{j}, p_{k}, p_{l}$ are the momenta of $\tilde{\chi}_{i}^{0}, \tilde{\chi}_{j}^{0}, \bar{f}$ and $f$, respectively. $D_{Z}^{\mu \nu}=\left[-g^{\mu \nu}+\left(p_{i}-p_{j}\right)_{\nu}\left(p_{i}-p_{j}\right)_{\mu} / m_{Z}^{2}\right] /\left[\left(p_{i}-p_{j}\right)^{2}-m_{Z}^{2}+\right.$ $\left.i m_{Z} \Gamma_{Z}\right]$ and $D_{S^{0}}=1 /\left[\left(p_{i}-p_{j}\right)^{2}-m_{S^{0}}^{2}+i m_{S^{0}} \Gamma_{S^{0}}\right]\left(S^{0}=h^{0}, H^{0}, A^{0}\right)$ are the propagators. The couplings are given by

$$
\begin{aligned}
O_{i j}^{L \prime \prime} & =\frac{1}{2 \cos \theta_{W}}\left[\left(N_{i 4} N_{j 4}-N_{i 3} N_{j 3}\right) \cos 2 \beta-\left(N_{i 3} N_{j 4}+N_{j 3} N_{i 4}\right) \sin 2 \beta\right] \\
L_{f} & =I_{f}^{3}-e_{f} \sin ^{2} \theta_{W}, \\
R_{f} & =-e_{f} \sin ^{2} \theta_{W}, \\
a_{j i}^{h^{0}} & =q_{j i} \sin \alpha+s_{j i} \cos \alpha, \\
a_{j i}^{H^{0}} & =-q_{j i} \cos \alpha+s_{j i} \sin \alpha, \\
a_{j i}^{A^{0}} & =-q_{j i} \sin \beta+s_{j i} \cos \beta, \\
q_{j i} & =\frac{1}{2 \cos \theta_{W}}\left[\left(N_{i 3} \cos \beta+N_{i 4} \sin \beta\right) N_{j 2}+\left(N_{j 3} \cos \beta+N_{j 4} \sin \beta\right) N_{i 2}\right] \\
s_{j i} & =\frac{1}{2 \cos \theta_{W}}\left[\left(N_{i 4} \cos \beta-N_{i 3} \sin \beta\right) N_{j 2}+\left(N_{j 4} \cos \beta-N_{j 3} \sin \beta\right) N_{i 2}\right] \\
a_{f}^{h^{0}} & =\frac{m_{f} \sin \alpha}{2 m_{W} \cos \beta}, \quad a_{f}^{H^{0}}=\frac{-m_{f} \cos \alpha}{2 m_{W} \cos \beta},
\end{aligned}
$$




$$
a_{f}^{A^{0}}=\frac{m_{f} \tan \beta}{2 m_{W}}(f=b, \tau)
$$

where $I_{f}^{3}$ is the $3 r d$ component of the isospin, $e_{f}$ is the charge of the fermion in units of the positron, and $N_{i j}$ is the neutralino mixing matrix in the notation of [8],

The important point here is that the $Z$-boson only couples to the higgsino components of the neutralinos, Eq. (7). Therefore, for $|M| \ll|\mu|$ the $Z$ - $\tilde{\chi}_{2}^{0}-\tilde{\chi}_{1}^{0}$ coupling can be rather small and the Higgs boson exchange contribution can become important due to the $h^{0}-b-\bar{b}$ coupling. This implies that the products of $Z$-couplings $L_{b} O_{21}^{L \prime}$ and $R_{b} O_{21}^{L \prime}$ 'can be smaller than the product of Higgs couplings $a_{j i}^{h^{0}} a_{b}^{h^{0}}$ as is demonstrated in the next section.

\section{$3 \quad$ Numerical Results}

In our numerical analysis we have taken $\sin ^{2} \theta_{W}=0.2315, \alpha\left(m_{Z}\right)=1 / 128$, $m_{Z}=91.187 \mathrm{GeV}, m_{\tau}=1.777 \mathrm{GeV}, m_{b}=5 \mathrm{GeV}$, and $m_{t}=175 \mathrm{GeV}$. In the $b$ Yukawa couplings, Eqs. (15) and (16), we have taken the running $m_{b}$ mass according to the renormalization group equations [9]. Moreover, we have fixed $M_{\tilde{F}}=500 \mathrm{GeV}(F=E, L, D, Q, U), A_{i}=100 \mathrm{GeV}(i=\tau, b, t)$, $M=120 \mathrm{GeV}$, and $M^{\prime}=5 / 3 \tan ^{2} \theta_{W} M$. With this choice of parameters the sfermion exchange is suppressed relative to the $h^{0}$ exchange. For the radiative corrections to the $h^{0}$ and $H^{0}$ masses and their mixing angle $\alpha$ we use the formulae of Ref. [10]; for those to $m_{H^{+}}$we follow Ref. [11].']

Fig. 11 shows the ratios of couplings $\left(a_{21}^{h^{0}} a_{b}^{h^{0}}\right) /\left(L_{b} O_{21}^{L \prime \prime}\right)$ (full line) and $\left(a_{21}^{h^{0}} a_{b}^{h^{0}}\right) /\left(R_{b} O_{21}^{L \prime \prime}\right)$ (dashed line) as a function of $\mu$ for $\tan \beta=4$ and $m_{A^{0}}=$ $125 \mathrm{GeV}$. In the regions with gaugino-like $\tilde{\chi}_{1,2}^{0}(|\mu| \geq M)$ these ratios are

${ }^{1}$ Notice that [10, 11] have a sign convention for the parameter $\mu$ opposite to the one used here. 
always larger than 1 and the absolute value of $\left(a_{21}^{h^{0}} a_{b}^{h^{0}}\right) /\left(R_{b} O_{21}^{L \prime \prime}\right)$ goes up to 15.

In Fig. 2 we show the branching ratios of $\tilde{\chi}_{2}^{0} \rightarrow \tilde{\chi}_{1}^{0} f \bar{f}$ as a function of $\mu$ where $f$ denotes $\nu_{l}, e, \tau, u, d$, or $b$. The other parameters are as in Fig. 1 . The final state with $b \bar{b}$ clearly dominates for $|\mu|>M$ due to the dominance of the Higgs exchange. Also the $\tilde{\chi}_{1}^{0} \tau^{-} \tau^{+}$final state is enhanced compared to the $\tilde{\chi}_{1}^{0} l^{-} l^{+}(l=e, \mu)$ final states for the same reason. The branching ratio for $\tilde{\chi}_{2}^{0} \rightarrow \tilde{\chi}_{1}^{0} b \bar{b}$ has maxima near $\mu= \pm 500 \mathrm{GeV}$. This can be explained by the fact that the gaugino components of $\tilde{\chi}_{1,2}^{0}$ are quite generally rising with $|\mu|$ and are constant for $|\mu| \gtrsim 500 \mathrm{GeV}$, whereas the higgsino components are decreasing with $|\mu|$.

Fig. 3 shows the ratio $B R\left(\tilde{\chi}_{2}^{0} \rightarrow \tilde{\chi}_{1}^{0} f \bar{f}\right) / B R^{\prime}\left(\tilde{\chi}_{2}^{0} \rightarrow \tilde{\chi}_{1}^{0} f \bar{f}\right)[f=e, \mu, \tau, b]$ as a function of $\mu$, where in $B R\left(B R^{\prime}\right)$ we have included (neglected) the Higgs boson contributions to the partial decay widths. The $e$ and $\mu$ channels can be reduced by up to $20 \%$. The branching ratios for the $\tau$ and $b$ channels are enhanced. The maxima for the $\tau$ channel near $\mu= \pm 500 \mathrm{GeV}$ can be explained in the following way: $B R^{\prime}\left(\tilde{\chi}_{2}^{0} \rightarrow \tilde{\chi}_{1}^{0} \tau^{+} \tau^{-}\right)$has minima at these $\mu$ values due to a destructive $Z-\tilde{\tau}_{i}$ interference, and $B R\left(\tilde{\chi}_{2}^{0} \rightarrow \tilde{\chi}_{1}^{0} \tau^{+} \tau^{-}\right)$is enhanced due to Higgs exchange.

Fig. 4 shows the $\tan \beta$ dependence of $B R\left(\tilde{\chi}_{2}^{0} \rightarrow \tilde{\chi}_{1}^{0} f \bar{f}\right)$ for $\mu=-500 \mathrm{GeV}$. The rising of the $b \bar{b}$ and $\tau^{+} \tau^{-}$channels is mainly due to the increase of the $b$ - and $\tau$ - Yukawa couplings. We also show in Fig. 5 the $\tan \beta$ dependence of the branching ratios for the $\tilde{\chi}_{1}^{+}$decays. Note that $B R\left(\tilde{\chi}_{1}^{+} \rightarrow \tilde{\chi}_{1}^{0} \tau^{+} \nu_{\tau}\right)$ is nearly twice $B R\left(\tilde{\chi}_{1}^{+} \rightarrow \tilde{\chi}_{1}^{0} e^{+} \nu_{e}\right)$ for large $\tan \beta$. The maximum (minima) of the $B R\left(\tilde{\chi}_{1}^{+} \rightarrow \tilde{\chi}_{1}^{0} q \bar{q}^{\prime}\right)\left(B R\left(\tilde{\chi}_{1}^{+} \rightarrow \tilde{\chi}_{1}^{0} e^{+} \nu_{e}\right)\right.$ and $\left.B R\left(\tilde{\chi}_{1}^{+} \rightarrow \tilde{\chi}_{1}^{0} \tau^{+} \nu_{\tau}\right)\right)$ at $\tan \beta \sim 10$ is due to a positive $W-\tilde{q}$ (negative $W-\tilde{l}$ and $W-\tilde{\tau}$ ) interference.

The $m_{A^{0}}$ dependence of the $\tilde{\chi}_{2}^{0}$ decay branching ratios is shown in Fig. 6 . 
As can be seen the Higgs boson exchange is important for $m_{A^{0}} \lesssim 200 \mathrm{GeV}$ and decreases for larger $m_{A^{0}}$. This is because $|\sin \alpha|$ decreases with increasing $m_{A^{0}}$ leading to a reduction the of $h^{0} b \bar{b}$ coupling [Eq. (15)].

In Fig. 7 we show the dependence of the $\tilde{\chi}_{2}^{0}$ branching ratios on the $U(1)$ gaugino mass parameter $M^{\prime}$ taking $M=500 \mathrm{GeV}, \mu=500 \mathrm{GeV}$, and $\tan \beta=4$. This dependence is rather weak except for $M^{\prime} \sim M$ where $m_{\tilde{\chi}_{2}^{0}} \sim m_{\tilde{\chi}_{1}^{+}} \sim m_{\tilde{\chi}_{1}^{0}}$

\section{Summary}

In this paper we have shown that the Higgs boson exchange contributions to three-body decay modes of the second lightest neutralino can be very impor$\operatorname{tant}$ even if $\tan \beta$ is small. This is valid for scenarios where the sfermions are heavy $\left(\gg m_{Z}\right)$ and $m_{A^{0}}$ is not too heavy $\left(m_{A^{0}} \lesssim 200 \mathrm{GeV}\right)$. This affects the signatures for $\tilde{\chi}_{2}^{0}$ decays at LEP, FNAL, LHC, an $e^{+} e^{-}$linear collider, and a muon collider. Most studies have been done within the minimal supergravity model $\left[4,12\right.$. A feature of this model is that $m_{A^{0}}$ is in general large and consequently $|\sin \alpha|$ relatively small, implying a small $h^{0}-b \bar{b}$ coupling for small $\tan \beta$. In a more general framework this is not necessarily true and the Higgs boson exchange contribution could be much larger.

\section{Acknowledgments}

This work was supported by the "Fonds zur Förderung der wissenschaftlichen Forschung" of Austria, project No. P13139-PHY. W.P. was supported by Spanish 'Ministerio de Educacion y Cultura' under the contract SB97-BU0475382. M. Kleander's help in checking some parts of the computer program is grate- 
fully acknowledged.

\section{References}

[1] see, for instance, A. Bartl, H. Fraas, and W. Majerotto, Nucl. Phys. B278, 1 (1986); A. Bartl, W. Majerotto, N. Oshimo and S. Stippel, Z. f. Phys. C47, 235 (1990); H. Baer and X. Tata, Phys. Rev. D47, 2739 (1993); H. Baer, C. Chen, C. Kao, and X. Tata, Phys. Rev. D52, 1565 (1995); S. Ambrosanio and B. Mele, Phys. Rev. D52, 3900 (1995), Phys. Rev. D53, 2541 (1996); M.M. Nojiri and Y. Yamada, Phys. Rev. D60, 015006 (1999), G. Moortgat-Pick, H. Fraas, A. Bartl, and W. Majerotto, hep-ph/9903220, to be published in Eur. Phys. J. C.

[2] H. Baer et al., Phys. Rev. Lett. 79, 986 (1997); Phys. Rev. D58, 075008 (1998).

[3] J.F. Gunion and H.E. Haber, Nucl. Phys. B272, 1 (1986); J.F. Gunion, H.E. Haber, G.L. Kane, and S. Dawson, The Higgs Hunters Guide, Addison-Wesley (1990).

[4] D. Treille, Invited talk at the $29^{\text {th }}$ Int. Conf. on High Energy Physics, Vancouver, Canada, 1998.

[5] S. Ambrosanio et al., Proc. of the Workshop 'Physics at LEP2', CERN 96-01, Vol. 1, p. 463, conveners G.F. Giudice, M.L. Mangano, G. Ridolfi, and R. Rückl, eds. G. Altarelli, T. Sjöstrand, and F. Zwirner.

[6] H. Baer, K. Hagiwara and X. Tata, Phys. Rev. D35, 1598 (1987); R. Arnowitt and P. Nath, Mod. Phys. Lett. A2, 331 (1987); H. Baer and 
X. Tata, Phys. Rev. D47, 2739 (1993); T. Kamon, J. Lopez, P. McIntyre and J. T. White, Phys. Rev. D50, 5676 (1994); H. Baer, C. Kao and X. Tata Phys. Rev. D48 (1993) 5175; H. Baer, C-H. Chen, C. Kao and X. Tata Phys. Rev. D52 (1995) 1565; S. Mrenna, G. Kane, G. D. Kribs and J. D. Wells, Phys. Rev. D53, 1168 (1996); H. Baer, C-H. Chen, F. Paige and X. Tata, Phys. Rev. D54 (1996) 5866; V. Barger, C. Kao, T.-J. Li, Phys. Lett. B 433, 328 (1998); V. Barger and C. Kao, hepph/9811489; H. Baer, M. Drees, F. Paige, P. Quintana, X. Tata, hep$\mathrm{ph} / 9906233$.

[7] F. Abe et al., the CDF collaboration, Phys. Rev. Lett. 76 (1996) 2228; S. Abachi et al., the D $\emptyset$ collaboration, Phys. Rev. Lett. 76 (1996) 4307.

[8] A. Bartl, H. Fraas, W. Majerotto and N. Oshimo, Phys. Rev. D40, 1594 (1989).

[9] Drees and Hikasa, Phys. Lett. B240, 455 (1990). Erratum, ibidem B262, 497, (1991).

[10] J. Ellis, G. Ridolfi, and F. Zwirner, Phys. Lett. B 262 (1991) 477.

[11] A. Brignole, Phys. Lett. B 277 (1992) 313.

[12] For reviews see Report of the mSUGRA Group in 'Proceedings of the Fermilab Tevatron Run II Workshop on SUSY/Higgs'; E. Accomando et al., Phys. Rep. 299, 1 (1998); S. Mrenna et al., Proc. of the 1996 DPF/DPB Summer Study on High Energy Physics, Snowmass, Colorado, Vol. 2, p. 681, D.G. Cassel, L. Trindle-Gennari, R.H. Siemann eds.; A. Bartl et al., ibidem, p. 693; M. Danielson et al., ibidem, p. 720. 


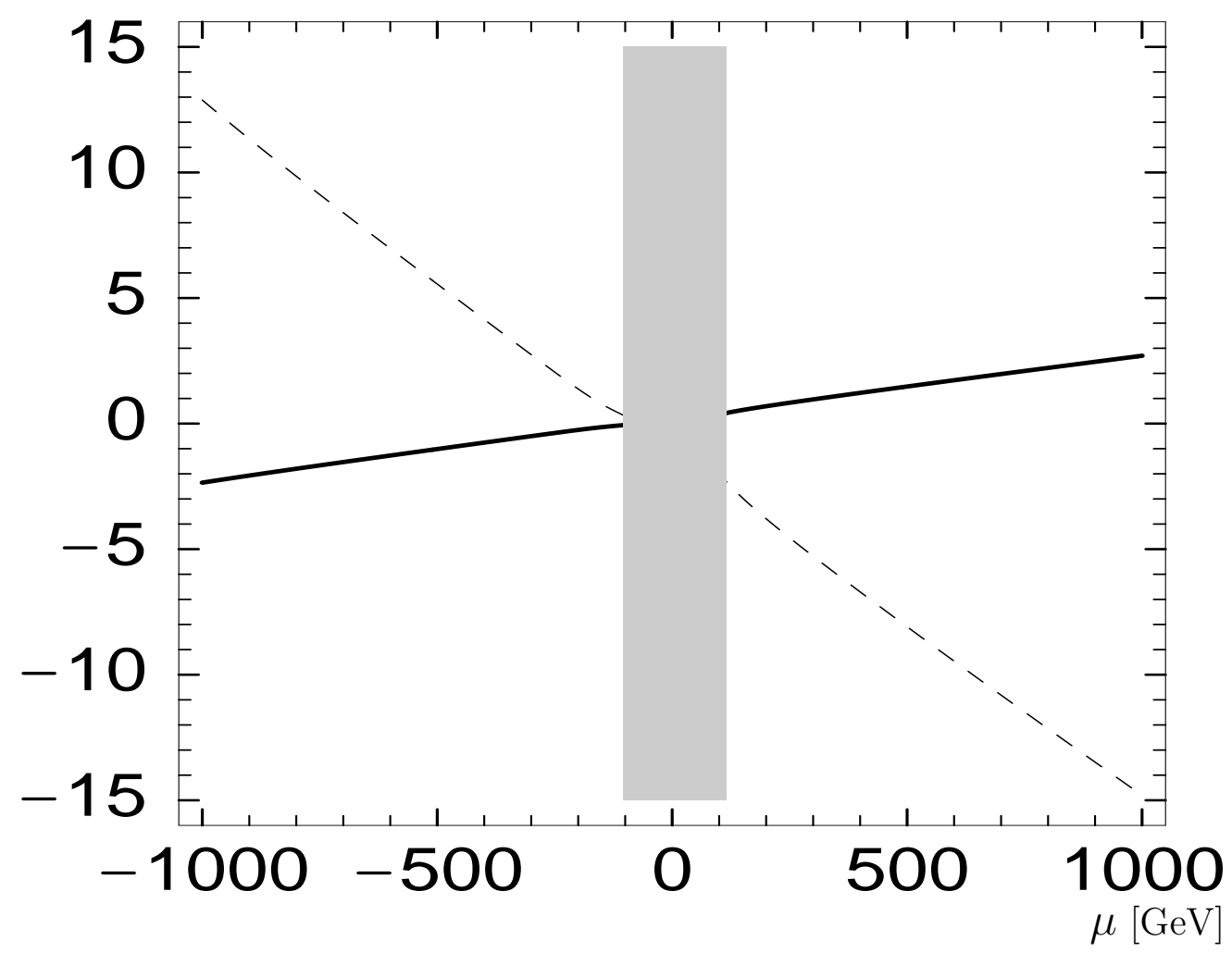

Figure 1: Ratios of couplings $\left(a_{21}^{h^{0}} a_{b}^{h^{0}}\right) /\left(L_{b} O_{21}^{L \prime \prime}\right)$ (full line) and $\left(a_{21}^{h^{0}} a_{b}^{h^{0}}\right) /$ $\left(R_{b} O_{21}^{L \prime}\right)$ (dashed line) as a function of $\mu$ for $\tan \beta=4$ and $m_{A^{0}}=125 \mathrm{GeV}$. The other parameters are given in the text and the couplings are defined in Eqs. (7) - (15). The grey area will be covered by LEP2 $\left(m_{\tilde{\chi}_{1}^{+}} \leq 95 \mathrm{GeV}\right)$. 


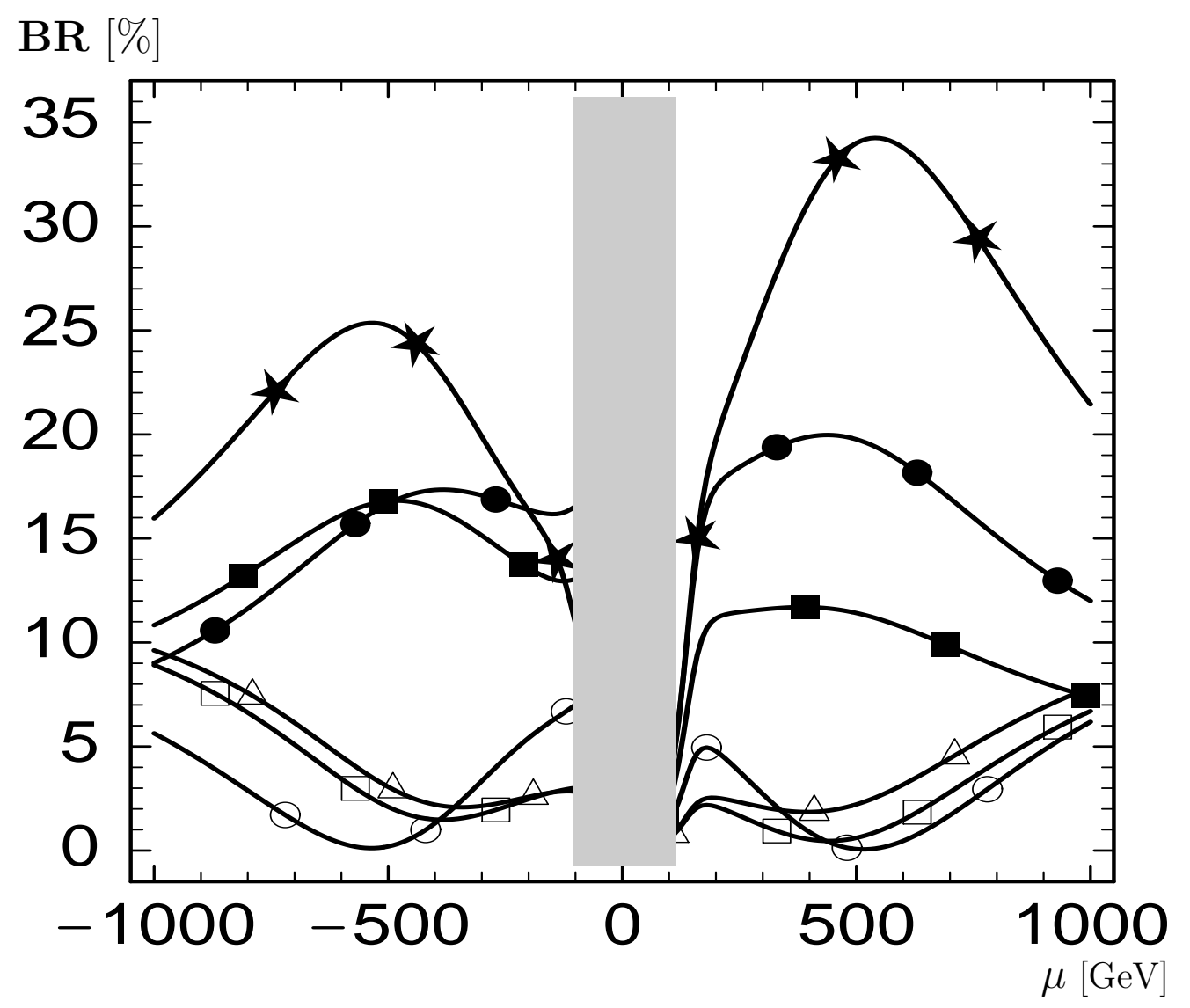

Figure 2: Branching ratios of $\tilde{\chi}_{2}^{0}$ as a function of $\mu$ for $\tan \beta=4$ and $m_{A^{0}}=$ $125 \mathrm{GeV}$. The other parameters are given in the text. The graphs correspond to following transitions: $\circ \tilde{\chi}_{2}^{0} \rightarrow \tilde{\chi}_{1}^{0} \nu_{l} \nu_{l}(l=e, \mu$, or $\tau), \square \tilde{\chi}_{2}^{0} \rightarrow \tilde{\chi}_{1}^{0} l^{+} l^{-}$ $(l=e$ or $\mu), \triangle \tilde{\chi}_{2}^{0} \rightarrow \tilde{\chi}_{1}^{0} \tau^{+} \tau^{-}, \tilde{\chi}_{2}^{0} \rightarrow \tilde{\chi}_{1}^{0} q \bar{q}(q=u$ or $c), \bullet \tilde{\chi}_{2}^{0} \rightarrow \tilde{\chi}_{1}^{0} q \bar{q}$ $(q=d$ or $s)$, and $\star \tilde{\chi}_{2}^{0} \rightarrow \tilde{\chi}_{1}^{0} b \bar{b}$. The grey area will be covered by LEP2 $\left(m_{\tilde{\chi}_{1}^{+}} \leq 95 \mathrm{GeV}\right)$. 


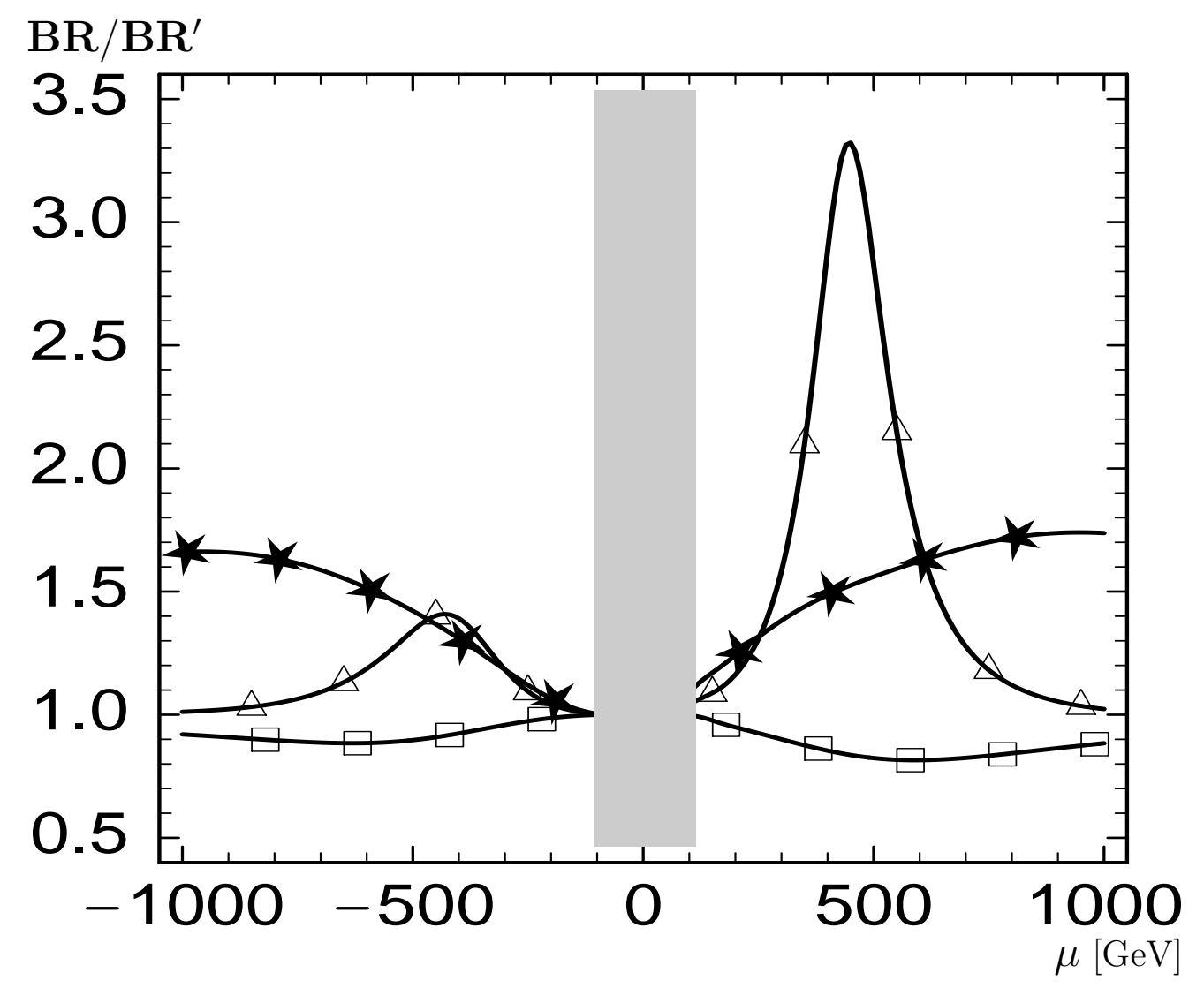

Figure 3: Ratio $B R\left(\tilde{\chi}_{2}^{0} \rightarrow \tilde{\chi}_{1}^{0} f \bar{f}\right) / B R^{\prime}\left(\tilde{\chi}_{2}^{0} \rightarrow \tilde{\chi}_{1}^{0} f \bar{f}\right)$ as a function of $\mu$ for $\tan \beta=4$ and $m_{A^{0}}=125 \mathrm{GeV}$. The other parameters are given in the text. In case of $B R\left(B R^{\prime}\right)$ we have included (neglected) the Higgs boson contributions to the partial decay widths. The graphs correspond to: $\square e$ or $\mu, \triangle \tau$, and $\star b$. 


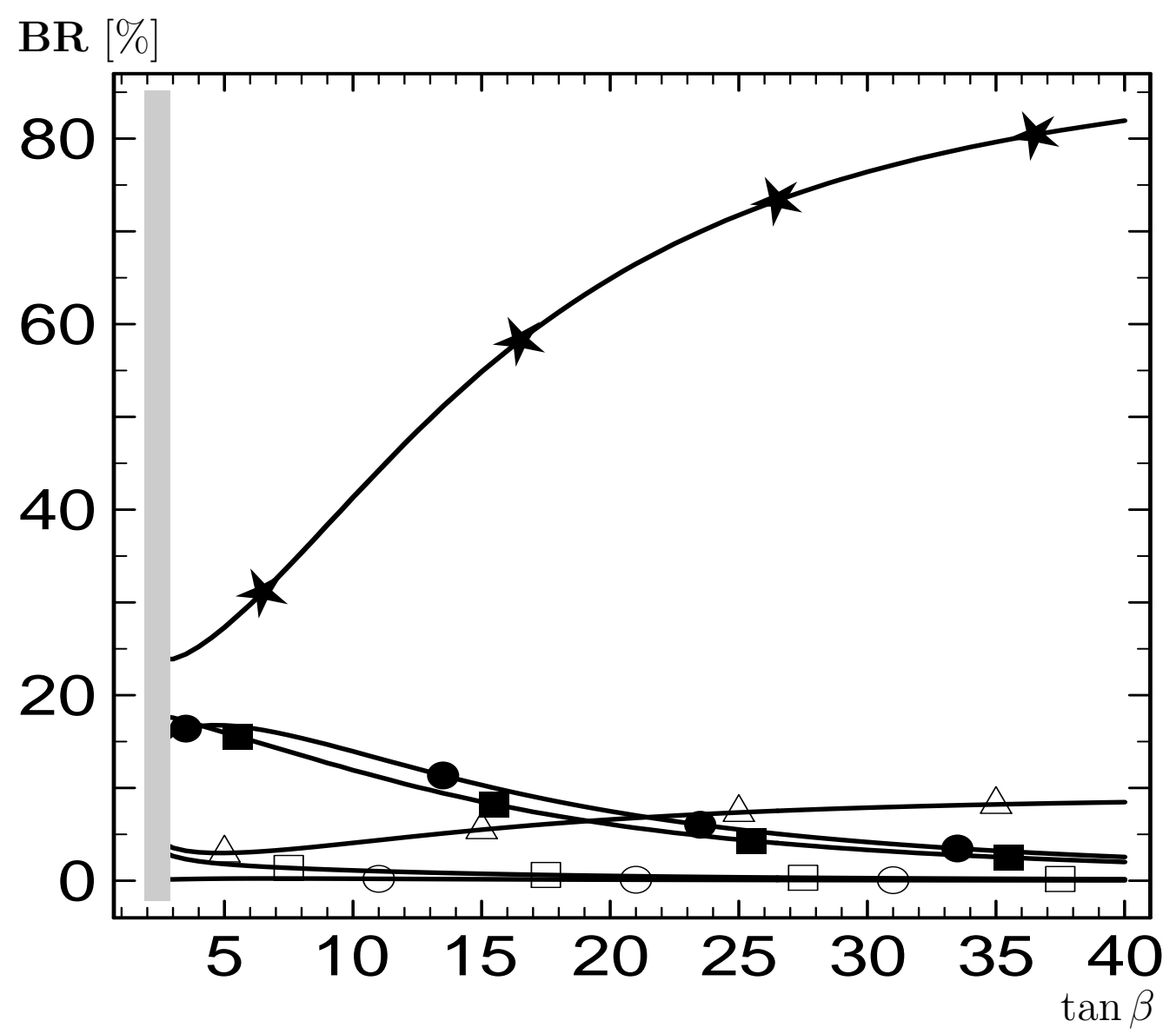

Figure 4: Branching ratios of $\tilde{\chi}_{2}^{0}$ as a function of $\tan \beta$ for $\mu=-500 \mathrm{GeV}$ and $m_{A^{0}}=125 \mathrm{GeV}$. The other parameters are given in the text. The graphs correspond to following transitions: $\circ \tilde{\chi}_{2}^{0} \rightarrow \tilde{\chi}_{1}^{0} \nu_{l} \nu_{l}(l=e, \mu$, or $\tau)$, $\square \tilde{\chi}_{2}^{0} \rightarrow \tilde{\chi}_{1}^{0} l^{+} l^{-}(l=e$ or $\mu), \triangle \tilde{\chi}_{2}^{0} \rightarrow \tilde{\chi}_{1}^{0} \tau^{+} \tau^{-}, \mathbf{\square} \tilde{\chi}_{2}^{0} \rightarrow \tilde{\chi}_{1}^{0} q \bar{q}(q=u$ or $c), \bullet \tilde{\chi}_{2}^{0} \rightarrow \tilde{\chi}_{1}^{0} q \bar{q}(q=d$ or $s)$, and $\star \tilde{\chi}_{2}^{0} \rightarrow \tilde{\chi}_{1}^{0} b \bar{b}$. In the gray area is $m_{h^{0}}<90 \mathrm{GeV}$. 


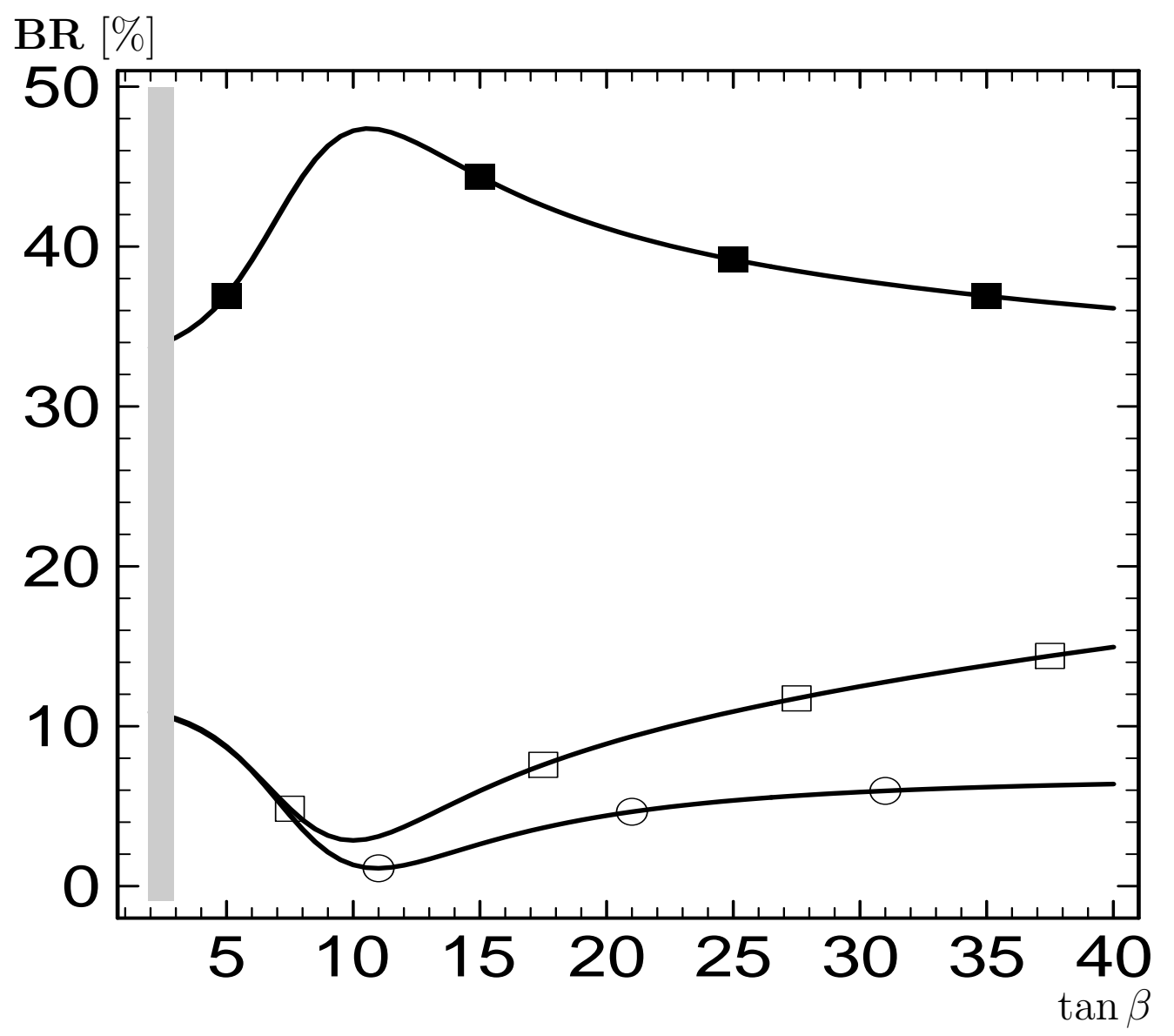

Figure 5: Branching ratios of $\tilde{\chi}_{1}^{+}$as a function of $\tan \beta$ for $\mu=-500 \mathrm{GeV}$ and $m_{A^{0}}=125 \mathrm{GeV}$. The other parameters are given in the text. The graphs correspond to following transitions: $\circ \tilde{\chi}_{1}^{+} \rightarrow \tilde{\chi}_{1}^{0} l^{+} \nu_{l}(l=e$ or $\mu)$, $\square \tilde{\chi}_{1}^{+} \rightarrow \tilde{\chi}_{1}^{0} \tau^{+} \nu_{\tau}$, and $\tilde{\chi}_{2}^{+} \rightarrow \tilde{\chi}_{1}^{0} q^{\prime} \bar{q}\left(\left(q^{\prime}, q\right)=(u, d)\right.$ or $\left.(c, s)\right)$. In the gray area is $m_{h^{0}}<90 \mathrm{GeV}$. 


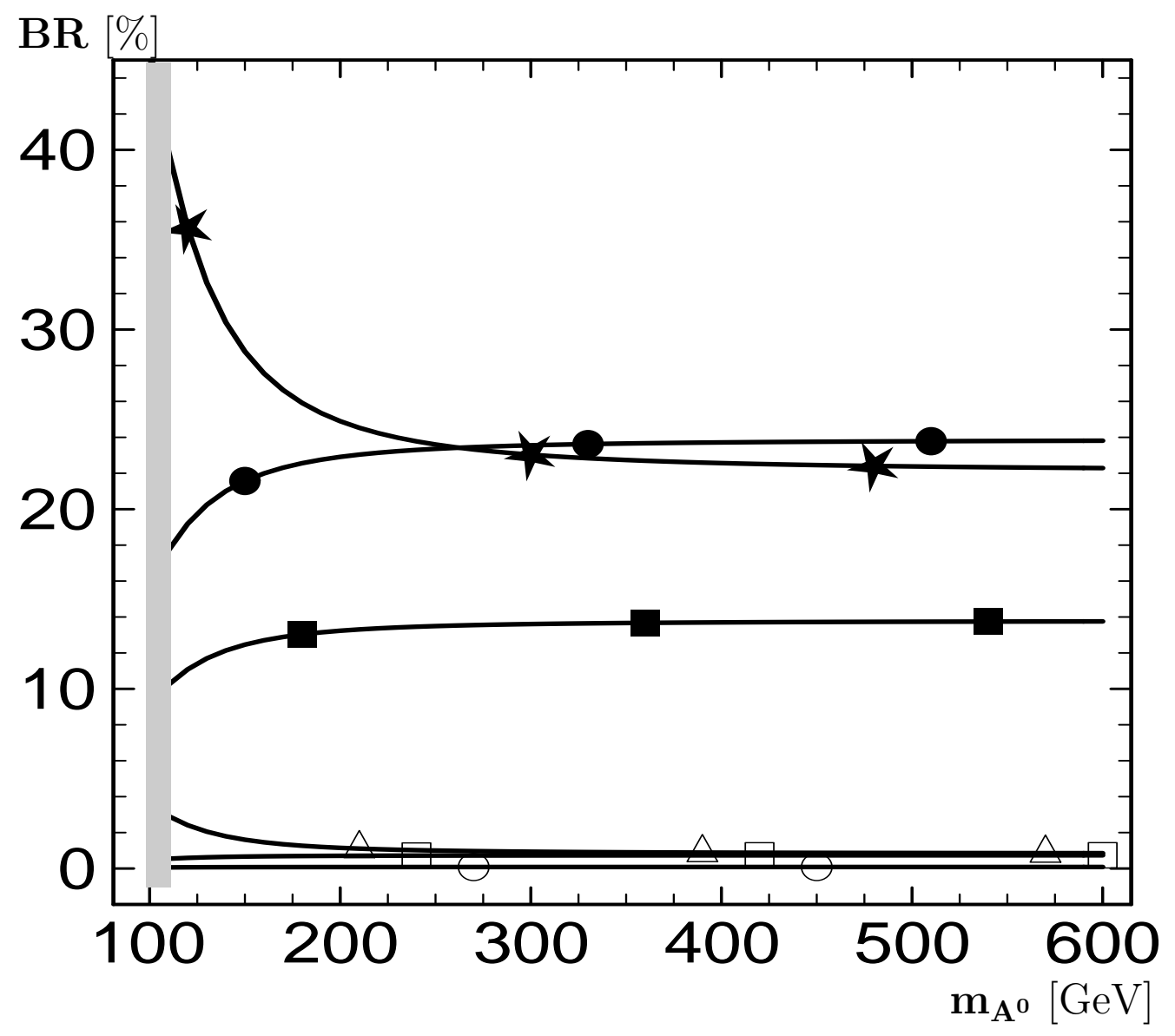

Figure 6: Branching ratios of $\tilde{\chi}_{2}^{0}$ as a function of $m_{A^{0}}$ for $\tan \beta=4$ and $\mu=500 \mathrm{GeV}$. The graphs correspond to following transitions: $\circ \tilde{\chi}_{2}^{0} \rightarrow \tilde{\chi}_{1}^{0} \nu_{l} \nu_{l}$ $(l=e, \mu$, or $\tau), \square \tilde{\chi}_{2}^{0} \rightarrow \tilde{\chi}_{1}^{0} l^{+} l^{-}(l=e$ or $\mu), \triangle \tilde{\chi}_{2}^{0} \rightarrow \tilde{\chi}_{1}^{0} \tau^{+} \tau^{-}, \mathbf{\square} \tilde{\chi}_{2}^{0} \rightarrow \tilde{\chi}_{1}^{0} q \bar{q}$ $(q=u$ or $c), \bullet \tilde{\chi}_{2}^{0} \rightarrow \tilde{\chi}_{1}^{0} q \bar{q}(q=d$ or $s)$, and $\star \tilde{\chi}_{2}^{0} \rightarrow \tilde{\chi}_{1}^{0} b \bar{b}$. In the gray area is $m_{h^{0}}<90 \mathrm{GeV}$. 


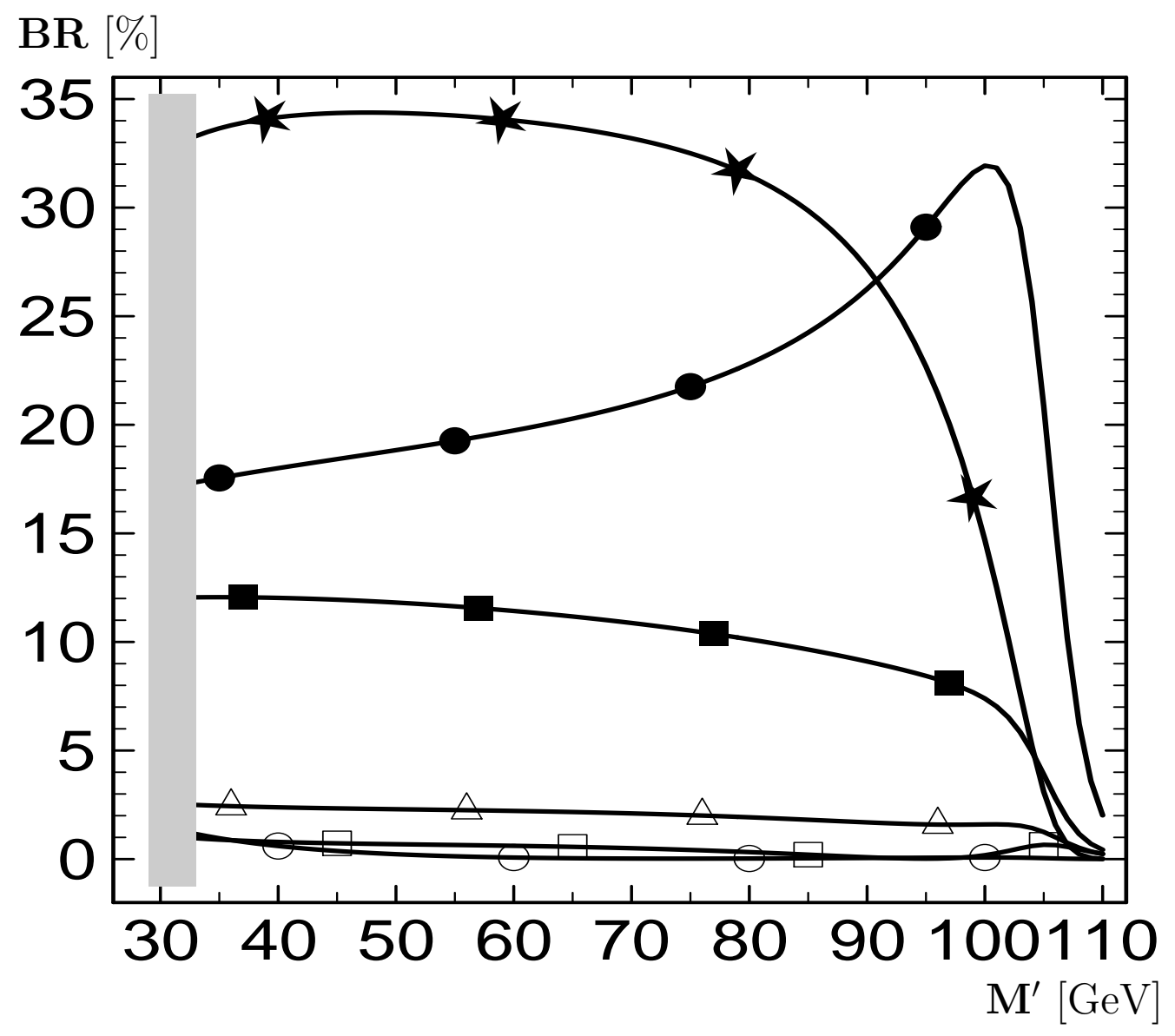

Figure 7: Branching ratios of $\tilde{\chi}_{2}^{0}$ as a function of $M^{\prime}$ (in $\mathrm{GeV}$ ) for $M_{2}=$ $120 \mathrm{GeV}, \mu=500 \mathrm{GeV}, \tan \beta=4$, and $m_{A^{0}}=125 \mathrm{GeV}$. The other parameters are given in the text. The graphs correspond to following transitions: $\circ \tilde{\chi}_{2}^{0} \rightarrow \tilde{\chi}_{1}^{0} \nu_{l} \nu_{l}(l=e, \mu$, or $\tau), \square \tilde{\chi}_{2}^{0} \rightarrow \tilde{\chi}_{1}^{0} l^{+} l^{-}(l=e$ or $\mu), \triangle \tilde{\chi}_{2}^{0} \rightarrow \tilde{\chi}_{1}^{0} \tau^{+} \tau^{-}$, - $\tilde{\chi}_{2}^{0} \rightarrow \tilde{\chi}_{1}^{0} q \bar{q}(q=u$ or $c), \bullet \tilde{\chi}_{2}^{0} \rightarrow \tilde{\chi}_{1}^{0} q \bar{q}(q=d$ or $s)$, and $\star \tilde{\chi}_{2}^{0} \rightarrow \tilde{\chi}_{1}^{0} b \bar{b}$. In the gray area is $m_{\tilde{\chi}_{1}^{0}}<30 \mathrm{GeV}$. 